\title{
Caracterización de las Organizaciones No Gubernamentales: El caso de los municipios Maracaibo y San Francisco
}

Ortiz, Rosa E.*

\section{Resumen}

El estudio de las Organizaciones No Gubernamentales estuvo dirigido a caracterizar la diversidad de grupos y asociaciones que bajo el termino genérico de ONG's existen en los municipios Maracaibo y San Francisco, los más poblados y relevantes en la vida política, económica, social y cultural del estado Zulia-Venezuela. Se planteó una investigación exploratoria y descriptiva. El resultado permitió reconocer a) Existe un crecimiento sostenido de las ONG en estos municipios: b) los sectores con mayor concentración son el de salud; educación; deporte y recreación; arte y cultura. c) Las instituciones que promueven más organizaciones son la iglesia y el Estado, lo que supone es este último caso una reducción de las funciones que le corresponden, además de la minimización de costos y conflictos laborales. d) Ambivalencia en la orientación de su funcionamiento: Algunas ONG's implementan prácticas próximas a la mercantilización de los servicios con lo cual legitiman estrategias del modelo económico neoliberal mientras otras contribuyen a fortalecer la democratización de los servicios públicos. Se concluye que el crecimiento y características de las ONGs en estos municipios estuvo vinculado en los noventa y comienzos de la década de 2000 por la aplicación de las políticas públicas neoliberales.

Palabras clave: ONGs, tercer sector, espacio público, municipio.

\section{Characterization of Non-Governmental Organizations: The Case of the Maracaibo and San Francisco Municipalities}

\section{Abstract}

This study of non-governmental organizations was directed towards a characterization of the diversity of groups and associations grouped under the generic term NGO that exist in the Mu-

Recibido: 04-01-31. Aceptado: 05-09-09

Investigadora del Centro de Estudios de la Empresa de la Universidad del Zulia. Licenciada en Trabajo Social (UCV), Magíster en Filosofía (LUZ) , con Especialidad en Dirección y Gestión Pública Local (IZEPES). E-mail: rortiz74@cantv.net 
nicipalities of Maracaibo and San Francisco, which are the more relevant populations in the political, economic, social and cultural life of Zulia State, Venezuela. A descriptive-exploratory study was proposed. The results indicated that a) there is sustained growth in the NGOs in these municipalities: b) the sectors with greater NGO concentration are health, education, sports, recreation, art and culture, c) the institutions that promote more organizations are the church and the state, which in the second case is an attempt to reduce the functions which correspond to the state, as well as to minimize labour costs and conflicts, d) There is ambivalence in the orientation of the functioning of NGOs. Some NGOs implement semi-mercantile activities in which they legitimize strategies within the neo-liberal economic model, while other groups strengthen the democratization of public services. The conclusion is that the growth and characterization of NGOs in these municipalities was related to the application of neo-liberal public policy during the 1990s and the beginning of the decade 2000 .

Key words: NGOs, third sector, public space, municipalities.

\section{Introducción}

El actual interés por el estudio de las Organizaciones No Gubernamentales está vinculado a la multiplicación y diversificación de grupos y asociaciones que, bajo el término genérico de ONG's, han venido desarrollando múltiples actividades, programas y proyectos para dar respuesta a los problemas sociales que atendidos de manera insuficiente antes de la implementación de las políticas de ajuste fiscal, se agudizan después de la reorientación hacia el modelo económico neoliberal. Es importante para su reconocimiento como actores sociopolíticos autónomos registrar sus prácticas y tipo de intervención, con lo cual se demostrará el crecimiento y la continuidad de las tendencias asociativas y espacios de participación.

En Venezuela, a diferencia de otros países latinoamericanos, el auge de las ONG's ha sido producto y expresión del agotamiento de los mecanismos de representatividad política del modelo rentista petrolero -caracterizado por el predominio de relaciones clientelares- que obstaculizaron las tendencias asociativas de la sociedad. De allí que se haga necesario referir, como antecedente de este estudio, la materialización de los procesos de cambios sociopolíticos que se han desarrollado en el país, donde un elemento central ha sido el redimensionamiento del Estado, a partir de los años 90 , cuando las relaciones económicas neoliberales impuestas provocaron la profundización de la exclusión y polarización social mientras el discurso político oficial ofrecía la ampliación del espacio democrático. No puede haber ampliación de la democracia en niveles extremos de pobreza y exclusión; ésta es la negación de derechos fundamentales, en consecuencia, es lo opuesto a la idea de nuevos actores y de nuevos escenarios.

En la actualidad es crecientemente cuestionada la atribución de un cúmulo de virtudes intrínsecas a las ONG's, pues no sólo se conocen experiencias de organizaciones cuyos procesos toman la forma de movimientos democratizadores, sino también otras que dirigen su proceso hacia la despolitización y desmovilización de las asociaciones populares y contribu- 
yen a legitimar los procesos de privatización y retraimiento del Estado en la atención de lo social.

En el presente artículo mostramos los resultados de la investigación realizada -en los Municipios Maracaibo y San Francisco, del estado Zulia- con el objetivo de identificar y clasificar las Organizaciones No Gubernamentales (ONG's) de esos municipios. A pesar de la importancia que han adquirido en el país al inicio de este estudio -año 2001- aún se sabe poco sobre ellas, pues son escasos los estudios nacionales y regionales.

La investigación se define como exploratoria y descriptiva. Exploratoria, porque se busca identificar la existencia, funcionamiento y tendencias que en su desarrollo han tenido las ONG's en estos municipios. Al inicio del proceso de estudio -año 2001- los datos ofrecidos tanto en la Gobernación del estado como en las Alcaldías, sobre estas organizaciones, eran escasos, repetitivos y referidos sólo a listas con nombres, teléfonos y dirección ${ }^{1}$. Este estudio es descriptivo porque se pretende además identificar las siguientes características: Ubicación espacial, ubicación sectorial, instituciones que las promueven, población objetivo, organización y permanencia y sus relaciones.

La investigación implicó la revisión de material bibliográfico para la delimitación del referente teórico-conceptual; la consulta de otras fuentes documentales y hemerográficas y la aplicación de un cuestionario dirigido al personal directivo de las ONG's. Se identificaron 165 ONG's del Portafolio Social ${ }^{2}$, correspondientes a Maracaibo y San Francisco, de las cuales se excluyeron las siguientes organizaciones: Las Asociaciones de Vecinos, las Cooperativas, Asociaciones Civiles registradas como clubes sociales y/o deportivos, los Partidos Políticos y asociaciones civiles y fundaciones creadas por instituciones del Estado y erróneamente consideras como $\mathrm{ONGs}^{3}$, incluidas en el

1 En un trabajo de Rodríguez et al (2002) se presentan los resultados de un estudio a las ONG's de los Municipios de interés en este trabajo, con una visión distinta a la aquí presentada. Rodríguez.

2 Inventario de ONG's del país, elaborado por el Centro de Investigaciones en Ciencias Sociales (CISOR) y la Sociedad Civil Servicio de Apoyo Local (SOCSAL).

3 Las Asociaciones de Vecinos, aun cuando se definen como instancias democráticas que deben fomentar la organización y promover la participación de la comunidad, están claramente definidas y registradas por la Alcaldía, regidas legalmente. Las Cooperativas también están reguladas en una ley donde se establece con claridad el concepto de cooperativa y los requisitos a cumplir para su constitución, organización y funcionamiento; están inventariadas por la Superintendencia de Cooperativas. Las asociaciones civiles registradas como clubes sociales y/o deportivos, las hemos excluido porque en su constitución priva un interés particular más que general y, usualmente, no prestan servicios sociales. Forman parte de la esfera corporativa (Bresser y Cunill, 1998) que aunque no tienen fines de lucro están orientadas a defender los intereses de grupo o corporación. Ej. Club de Leones, Rotary Club. También excluimos a los Grupos religiosos que trabajan en centros catequísticos y en actividades pastorales, cuya orientación es fundamentalmente de carácter espiritual. Sin embargo, aquellas asociaciones promovidas desde la iglesia -evangélica y/o católica- para cumplir una actividad de servicio social, pres- 
mencionado Portafolio, reduciéndose nuestra población a 122 organizaciones, las cuales fueron cotejadas con otras fuentes ${ }^{4}$, lo que arrojó un total de 195 ONG's en los dos Municipios. El cuestionario fue aplicado a un $85 \%$ de estas organizaciones, para actualizar información del Portafolio Social y levantar información no existente.

\section{Contexto de Estudio}

Los municipios Maracaibo y San Francisco son los más poblados -concentran el $60 \%$ de los habitantes- del estado Zulia primero en importancia estratégica del país. Se caracterizan por la presencia de altos niveles de pobreza $(45,94 \%)$, muy bajo nivel de asociación y organización; donde se ubican las sedes administrativas del poder político estadal y las empresas estratégicas en el aporte económico (no sólo del Zulia sino del país), de importantes grupos sociales y culturales de la región (CONZUPLAN, 2000).

El Municipio Maracaibo está conformado por 18 parroquias, siendo éstas: Bolívar, Cacique Mara, Caracciolo Parra
Pérez, Cecilio Acosta, Cristo de Aranza, Coquivacoa, Chiquinquirá, Francisco E. Bustamante, Ildefonso Vásquez, Juana de Ávila, Luis Hurtado Higuera, Manuel Dagnino, Olegario Villalobos, Raúl Leoni, Santa Lucía, Venancio Pulgar, Antonio Borjas Romero y San Isidro. Tiene una superficie de $393 \mathrm{Km} 2$, con una población estimada de 1.449.771 habitantes que representa el $43,69 \%$ del total del Estado. Su actividad económica primordial es la terciaria, con gran concentración de empresas dedicadas a actividades comerciales y servicios en general, entre las que destaca PDVSA Occidente.

San Francisco pasó a constituirse en Municipio el 8 de marzo de 1995, cuando la Gobernación del Estado Zulia decide la división del Municipio Maracaibo (CONZUPLAN, 1996). El Municipio San Francisco posee seis parroquias: San Francisco, El Bajo, Domitila Flores, Los Cortijos, Francisco Ochoa y Marcial Hernández. Con una superficie de 164 $\mathrm{Km} 2$ con una población estimada en 416.483 habitantes; la economía se sustenta en actividades de los sectores secundarios y terciarios, donde destaca la

tadora de servicios a las comunidades o grupos específicos sí fueron incluidas. Ej. Fe y Alegría. Los partidos políticos: Aun cuando constituyen asociaciones cuyos miembros se reúnen, de acuerdo a estatutos y programas libremente acordados, para jugar un papel primordial como promotor de la participación ciudadana y articulador de las aspiraciones populares, en nuestra realidad han relegado este papel para constituirse en una simple maquinaria electoral, incapaz de interpretar apropiadamente las aspiraciones de la sociedad civil. Su actividad es regida por la Ley de Partidos Políticos, Reuniones Públicas y Manifestaciones. Las fundaciones y asociaciones civiles creadas por instituciones del Estado, son producto de decretos a distintos niveles de gobiernos y reglamentos de algunas instituciones como la Universidad del Zulia (LUZ), por ejemplo: Fundación República de los Muchos (de la Gobernación), A.C. Casa Mía (de la Alcaldía de Maracaibo) y Hábitat LUZ (de LUZ).

4 Gobernación del Estado, Alcaldía de Maracaibo, Alcaldía de San Francisco y Arquidiócesis de Maracaibo. 
actividad de petróleo y carbón, aunque existen experiencias exitosas en cultivos agrícolas menores: barbacoas (CONZUPLAN, 2000).

La división política ha diferenciado las formas de organización local, mas los estudios nos indican que comparten las mismas fortalezas y debilidades. Entre las fortalezas que se les reconocen podemos señalar: Su ubicación geográfica para intercambio comercial con Colombia y otras áreas andinas y del Caribe; población base de un mercado de productos y servicios de consumo masivo; servicios básicos para el funcionamiento de industrias, comercio y servicios; sede de instituciones gubernamentales y de sociedad civil organizada: Cámaras, ONG's, y Asociaciones; infraestructura de apoyo, vialidad, aeropuerto, puerto, telecomunicaciones, servicios de transporte; comercialización y capacidad hotelera; relaciones fronterizas; actividades administrativas y financieras de petroquímica, petroleras; sus debilidades están referidas a numerosos problemas como el incremento de los índices de pobreza: se sitúan entre las ciudades a nivel nacional que presenta mayores índices de pobreza con un $45,94 \%$; vulnerabilidad económica: actividades que generan poco valor agregado; finanzas públicas insuficientes; dispersión, anarquía y segregación urbana: un $60 \%$ de la superficie la ocupan los asentamientos urbanos precarios; deficiente organización y participación ciudadana; altos niveles de burocracia; servicios públicos deficitarios; deficiente formación de recursos humanos en áreas puntuales de producción (nuevos técnicos y universitarios); inexistencia de valores de disciplina y voluntad de trabajo a la innovación tecnológica; deficiente cultura ciudadana. Poca voluntad política a proyectos de mediano y largo plazo (Alcaldía de Maracaibo y LUZ, 1994. CONZUPLAN, 2000).

Este diagnostico institucional que reconoce a las ONG's como fortaleza refiere, sin embargo, bajo nivel de participación y organización ciudadana. En este mismo sentido resulta notable que ninguna oficina del sector gubernamental -regional y local- de cuenta de una información relevante e indicadora de la articulación de estas organizaciones en los planes de desarrollo de gobierno; sólo se manejan listas de ubicación -teléfonos y/o dirección- de aquéllas con las que mantienen relaciones de trabajo.

\section{Hacia una definición de las ONG's}

A finales de la década de los ochenta en Venezuela, con el agravamiento de la crisis económica, el proceso inflacionario y la incapacidad de generar suficientes recursos para atender los servicios públicos, ligado a la percepción de la administración pública concebida como ineficiente y corrupta, se inicia un conjunto de reformas y programas alentados por los organismos multilaterales como el Banco Mundial (BM) y el Fondo Monetario Internacional (FMI), entre las cuales se destaca la incorporación de Organismos No Gubernamentales en ciertas dimensiones de la política social.

Desde entonces se comenzó a generar un ambiente nacional e internacional favorable a las ONG's tendiente a fortalecer y apoyar el crecimiento de estas organizaciones, las cuales pueden plani- 
ficar y ejecutar programas sociales con dos concepciones: una fundamentalmente asistencialitas dirigidas a disminuir los efectos del impacto de las políticas de ajuste y, otra de tendencia alternativa orientada a contribuir a la organización y fortalecimiento de actores que puedan incidir en las políticas públicas. Es así que encontramos que en Venezuela, algunas ONG's han orientado su trabajo -con amplio apoyo del gobierno norteamericanoal ámbito de la política en la defensa del modelo neoliberal, como son los casos de Queremos Elegir y Súmate, que provienen de las asociaciones vecinales de clase media de la ciudad de Caracas.

Algunas organizaciones de la sociedad se constituyen como personas jurídicas de derecho privado bajo la forma de asociaciones civiles o fundaciones identificándolas comúnmente como "Organizaciones No Gubernamentales", término que agrupa un muy variado tipo de asociaciones tan amplio, complejo y heterogéneo entre sí como: hospitales, escuelas, clubes deportivos, centros de prestación de servicios comunales, de formación profesional, culturales, recreativos y muchos otros.

Ante esta variedad de organizaciones se dificulta establecer una definición única que capte los rasgos más importantes de este actor social emergente. Las siglas ONG, aunque han ganado un reconocimiento mayoritario, sólo recogen la connotación negativa en el sentido de no ser gubernamentales, pero no precisan las características de ese conjunto tan heterogéneo, al cual además también se le identifica de formas tan diversas como "sector no lucrativo", "sociedad civil", "sector del voluntariado" y "organizaciones de desarrollo social", entre otras.

Francisco Covarrubias (1992) define las ONG's como instituciones privadas de la sociedad civil -vinculadas al quehacer social- que operan como cuerpos intermedios entre el Estado y la comunidad de base, algunas consideradas alternativas por cuanto sus programas de acción son realizados en contraposición a los emprendidos por el gobierno. En una línea similar encontramos a Mario Padrón (citado por Lavadenz, 1992) quien las define como organizaciones privadas sin fines de lucro, públicamente registradas o con estatus jurídico, cuya principal función es llevar a cabo proyectos de desarrollo que favorezcan a los sectores populares y por lo cual reciben soporte financiero, casi siempre de otras ONG's de países industrializados que operan en el campo internacional.

Estos autores coinciden con Vargas (1999) al señalar que dichas organizaciones están vinculadas al ámbito privado que demuestra interés por lo público, y así se distinguen del Estado y la colectividad. Contrario a esta posición Morales (1998) considera poco afortunado destacar el carácter privado de estas organizaciones, aun cuando estén orientadas hacia el interés público, por cuanto en la esfera privada se ubican empresas y corporaciones estatales que encuentran su lógica en la defensa de intereses particulares, económicos o sectoriales, comportándose como organizaciones de mercado; mientras en la esfera social pública es donde se encuentran los movimientos y las instituciones que, aun cuando formalmente privados, persiguen objetivos más del colectivo. 
Bresser y Cunill (1998) entienden lo público como lo que es de todos y para todos, opuesto tanto a lo privado -que está volcado al lucro o al consumo- como a lo corporativo -que está orientado a la defensa de los intereses sectoriales o grupales-. Dentro de lo público distingue lo público estatal y lo público no estatal, con lo cual se muestra que la responsabilidad de participar en la satisfacción de las necesidades colectivas de la sociedad no es un campo donde el Estado es única opción, ya que la esfera pública que se ocupa de la producción social tiende a ampliarse con la incorporación de estos nuevos actores.

Algunos autores (Cernea,1992; Covarrubias; 1992, Bresser y Cunill, 1998 y Morales, 1998) vinculan las ONG's al Tercer Sector por ser un espacio de participación y experimentación de nuevos modos de pensar y hacer sobre la realidad social, con una propuesta organizacional, una veces de carácter instrumental y otras veces política, que busca una estrategia alternativa -orientada por el interés general- ante los programas de acción ofrecidos por el Estado. A diferencia de esta propuesta, Defourney, (1994) plantea que el Tercer Sector está formado además por aquellas organizaciones que prestan servicios a sus miembros, como las mutuales, las cooperativas y las asociaciones profesionales.

Todos los autores señalados coinciden en reconocer una externalidad positiva a las ONG's ya que incorporan de entrada un cúmulo de virtudes entr las que podríamos señalar las siguientes: (Pro Mundi Vita Estudios, 1989; Salamón y Anheier,1999; Morales, 1998).
Altruismo: Por su particular relación para la prestación de servicios de solidaridad y responder a las necesidades de grupos vulnerables en áreas no atendidas por el sector gubernamental.

Autonomía frente al Estado: Son distintas e independientes de los gobiernos y auspician el desarrollo de una institucionalidad alternativa. No son parte integrante del aparato de gobierno nacional, regional o local, aun cuando reciban subsidios públicos no se ejerce sobre ellos ningún tipo de autoridad oficial, a excepción de la derivada de las funciones regulatorias.

Autogobierno: Las autoridades internas de los entes determinan por sí mismas las actividades que llevan a cabo, éstas no son determinadas por gobierno ni por una empresa privada.

Adhesión libre y voluntaria: Reúne personas por adhesión libre que establecen entre ellas relaciones de igualdad, sin fines de lucro, pues no reparten beneficios para el conjunto de titulares de la misma e incorporan aportes voluntarios de tiempo y dinero.

Institucionalización: Presentar rasgos de organización de sus actividades en torno a fines relativamente persistentes. Los grupos no pueden ser efímeros y sus miembros deben ser distinguibles de los no miembros Tener un registro que le dé personalidad jurídica.

Sin fines de lucro: Los entes no distribuyen beneficios ni dividendos; el superávit que arrojen los balances se revierte a favor de la misión institucional. Sus objetivos no se orientan a fines comerciales o a producir beneficios. De participación, puesto que son proveedoras 
de canales de participación, dando un aporte al empoderamiento social $y$, en particular, al desarrollo de capacidades y habilidades para que las propias comunidades intervengan en su propia conducción.

De participación: Son proveedoras de canales de participación, dando un aporte al empoderamiento social y, en particular, al desarrollo de capacidades y habilidades para que las propias comunidades intervengan en su propia conducción.

De acuerdo con estos criterios se pueden considerar ONG's las asociaciones profesionales, partidos políticos, iglesias, sindicatos, asociaciones de vecinos y cooperativas, tal como lo confirma las propuestas de clasificación de las ONG's presentadas por Gruson et al (1997) y González (1998), algunas de éstas registradas en el Portafolio Social, como señalamos en páginas anteriores.

\section{Antecedentes de las ONG's en Venezuela}

La densidad de Organizaciones No Gubernamentales y la presencia de las agencias de cooperación internacional han variado según los países. Su inserción y crecimiento fue inicialmente mayor en los países más pequeños y con mayores niveles de pobreza; mientras que los países más desarrollados con frecuencia son sede de las organizaciones financistas proveedoras de recursos. Por otra parte, la modalidad predominante de relación entre el Estado, los partidos políticos y organizaciones de la sociedad que prevalezcan, en cada país, dará la tendencia a su crecimiento y dinamismo.
En la década de los sesenta y setenta, en algunos de los países de Sur América el surgimiento de las ONG's coincide con diferentes movimientos sociales y nuevas formas de acción colectiva como alternativa al cierre de los tradicionales canales institucionales de expresión de demandas sociales por la instauración de dictaduras militares. En este período las ONG's desplegaron su actividad proporcionando apoyo humanitario a las víctimas de las dictaduras militares y denunciando las violaciones de los derechos humanos, lo cual creó una imagen favorable de las ONG's hasta en los movimientos de izquierda, considerándolas como parte del campo progresista (Petras, 2000).

Otro importante hecho para la difusión de estos movimientos fue la influencia de las Conferencias Episcopales efectuadas en Medellín, Colombia en 1968 y Puebla, México en 1979, las cuales expresaron el cambio de postura de la Iglesia católica hacia los pobres y contribuyó a ampliar la presencia de sectores progresistas de dicha iglesia, así como la creación y formación de organizaciones de base.

En la misma década de los años setenta, la influencia en foros internacionales de la voz de protesta social de grupos tradicionalmente excluidos -las mujeres, los negros- convertidos en movimientos sociales generaliza un debate sobre la discriminación y la lógica de la igualdad, lo cual promueve no sólo algunas transformaciones legales de reconocimiento social y político de ciertas violaciones a sus derechos sino las tendencias a organizar grupos de defensa de derechos sociales y políticos que se derivan de esos movimientos. 


\subsection{Década de los 50. La expresión de la iglesia católica durante la dictadura}

El surgimiento de las ONG's estuvo vinculado a las acciones de la Iglesia católica, principalmente a mediados de la década del 50 , bajo la dictadura de Pérez Jiménez -aun cuando desde 1927 se funda el Hogar de Ancianos San José de la Montaña en Maracaibo (Rodríguez et al, 2004). Para entonces se inicia la Organización "Fe y Alegría", con la fundación de la primera escuela de carácter popular en un barrio de Petare; la Fundación La Salle de Ciencias Naturales -dedicada a impartir educación técnica para el trabajo e investigaciones científicas relacionadas con las ciencias naturales y sociales- emprende sus primeras actividades; el hogar San Juan de Dios en Caracas y el Hogar Clínica San Rafael en Maracaibo inician sus actividades en el área de salud. Sin embargo, para ese momento no se da un reconocimiento ni crecimiento generalizado a este tipo de organizaciones, pues las relaciones Estado-Sociedad que dominaban en el país, y la bonanza económica no lo favorecía.

\subsection{Década de los 60. Consolidación de la democracia representativa: Dominación de los espacios públicos por los partidos políticos}

A finales de la década de los cincuenta e inicios de los sesenta - contrariamente a lo que sucede en la mayoría de los países latinoamericanos donde predominan las dictaduras- en Venezuela se inicia y consolida la democracia representativa, de afiliación socialdemócrata. Los partidos políticos y las elecciones se convierten en el canal casi exclusivo de mediación entre el Estado y la sociedad, instaurando un modelo de ciudadanía correspondiente al de militantes o simpatizantes de partido, que monopolizaban la vida colectiva y constituían el único canal de mediación entre la sociedad y el Estado, ejerciendo control e influencia en todos los ámbitos de la vida publica: organizaciones sindicales, vecinales, gremiales, culturales, científicas, económicas y sociales. Las elecciones eran el ritual quinquenal donde se le atribuía a los representantes elegidos la facultad de pronunciarse y decidir sobre todos los asuntos del Estado, donde sólo eran convocados los actores corporativos tradicionales: burguesía, militares y sindicalistas. Para otros actores los canales de participación eran de carácter clientelar como simpatizantes o militantes de partidos políticos y/o a través de protestas sociales. (Lander, 2000).

En este periodo se puso en marcha el proceso de modernización de corte desarrollista canalizado por el Estado y los partidos políticos, con exclusión de otros actores de la sociedad civil. Este modelo impulsó un crecimiento económico sostenido con un empobrecimiento y desigualdad creciente, pues durante la privilegiada situación financiera, la utilización del excedente contribuyó tanto a incrementar la concentración industrial como a producir un proceso de centralización de la propiedad y del capital industrial (Chossudovsky, 1979).

\subsection{Década de los 70. El boom petrolero y el adormecimiento de la sociedad civil}

La abundante y creciente renta petrolera generada entre los años sesenta y 
setenta en especial, generó tal cantidad de recursos en manos del Estado que reforzó su concepción como principal y único agente para la distribución de la renta e impulsador del proceso de modernización del país. Se crea entonces infraestructura, se incrementan los servicios públicos básicos, se invierte directamente en actividades productivas y se apoya la actividad privada, sustentado en lo que se ha llamado el modelo rentista petrolero de la economía venezolana (Lander, 2000). La abundancia de recursos manejados facilitaba establecer mecanismos de conciliación o cooptación con otros actores sociales, lo cual debilitaba la capacidad asociativa y organizativa de la población para incidir en las estructuras estatales y las políticas públicas.

\subsection{Década de los 80. Expresión de la crisis del modelo de ciudadanía impuesto. Repunte de formas alternativas de Participación}

A principio de la década de los ochenta se inicia el debilitamiento del modelo rentista petrolero, lo cual marcó el inicio de una profunda crisis estructural, entre cuyas causa podemos señalar: el endeudamiento externo que favorecía objetivamente al capital financiero internacional por la transferencia de capitales al exterior, el deterioro de los términos de intercambio en la política de exportaciones y la caída de los precios del petróleo, lo que disminuyó la capacidad del Estado para satisfacer el sistema de prebendas con el cual conciliaba las demandas heterogéneas de los distintos actores.

Como principal responsable de la profunda crisis se señala a la excesiva in- tervención estatal, ineficiencia, corrupción y clientelismo que predominaba en la administración pública. Se buscan soluciones en la reducción de la política, la tendencia es limitar el papel del Estado y el descrédito de los partidos políticos, alentando los discursos del fin de la política, con temas recurrentes como apolíticos, antipolítico, sustrayendo así, las decisiones de políticas económicas del debate político. Estas concepciones van en sintonía al pensamiento político neoliberal del fin de las ideologías y de la historia, cuando en la práctica sociopolítica se presentan líderes que con la reconducción del discurso político -que pasa por la aceptación del paquete- y pretensiones de antipolíticos, buscan espacios y reconocimiento para sustituir actores de la vieja política.

Para el año 1989 las opciones para enfrentar la crisis fiscal se orientaron por las recomendaciones del FMl y el BM sin la búsqueda de consulta $\mathrm{y}$, menos aun, de un mínimo de consenso en las fuerzas sociales tradicionales ni con las fuerzas emergentes del país, pero sin embargo, sí contó con las preferencias de los actores económicos más vinculados al comercio exterior y al sector financiero. El recetario impuesto por estos organismos internacionales, conocido como Consenso de Washington, incluía diez ingredientes de política económica:1) Disciplina fiscal, 2) Priorización del gasto público en áreas de alto retorno económico, 3) Reforma tributaria, 4) Tasa positivas de interés fijadas por el mercado, 5) Tipos de cambio competitivos y liberalización financiera, 6) Liberalización comercial, 7) Apertura de la inversión extranjera, 8) Privatizaciones, 9) Desregulación am- 
plia y 10) Protección a la propiedad privada (Vilas, 2000). Todas estas medidas se impulsaron como si se tratase exclusivamente de un asunto técnico, sin intento alguno de crear coaliciones ni consensos en torno a los cambios pretendidos. La reacción social a estas medidas en Venezuela fue por la vía popular, a través de la explosión social de febrero de 1989 , y extra-institucional por el intento de golpe de Estado de 1992, que contó con un amplio, aunque pasivo, apoyo popular (Lander, 2000).

La implementación de las recetas fue dirigida a desmontar los esquemas intervencionistas del Estado, transfiriéndoles activos y capacidades de decisión a los actores del mercado; se eliminan controles gubernamentales sobre la inversión, la fijación de precios, el crédito, las transacciones comerciales y financieras internas e internacionales, y se contrae el gasto social. Sin embargo, la intervención estatal aumenta pero cambian los actores que dirigen el Estado y la dirección de la intervención estatal.

Se favoreció el auge del capital financiero en detrimento de los sectores productivos cuyo poder de influencia en el Estado marcó una victoria del capital sobre la fuerza de trabajo expresada en el retroceso de la movilización y presión sindical. Todo ello implicó cambios en las condiciones formales de contratación, de la estabilidad se pasa a la flexibilización con lo cual hay restricciones para acceder a convenios o contratos en defensa de las condiciones de trabajo, además de la pérdida y debilitamiento de logros alcanzados duramente y por la lucha de la fuerza trabajadora durante largos años, como por Ej. Antigüedad y prestaciones sociales.

Esta reestructuración tuvo como consecuencia un conjunto de manifestaciones de deterioro social que fueron más allá del momento inicial del ajuste y aun hoy se mantienen tales como: Fragmentación del mercado de trabajo con alta tasa de desempleo, subempleo y sobre ocupación; deterioro de las remuneraciones reales, y aumento de la población en condiciones de pobreza y pérdida de acceso a los sistemas de servicios públicos. La atención de las necesidades sociales que siempre se vio insuficientemente resuelta, adquiere una magnitud mas dramática con el inicio del proceso privatizador, especialmente en los sectores populares y clase media, donde aumenta la población en pobreza y empobrecida.

Todas estas condiciones aunadas también a la influencia de los movimientos sociales desarrollados en otros lugares del continente, propicia la presencia de grupos organizados en el espacio público reclamando participación e inclusión en defensa de sus derechos. Destaca, entre otros, la actuación de los grupos feministas los cuales, con el apoyo desde el Estado a través del Ministerio para el Desarrollo de la Mujer, impulsan un fuerte movimiento social que permitió la modificación de la Reforma Parcial del Código Civil, con lo cual se da un importante avance en el reconocimiento social, político y jurídico de ciertas violaciones a los derechos humanos; igualmente se forman en varias ciudades del país las Casas de la Mujer. También se inicia el movimiento vecinal, el cual comenzó vinculado con los sectores de clase media, y se 
identificó más fácilmente con la representación de sus intereses como grupo que con en el patrón de movimiento social equivalente a actor generador de cambio social (Ramos, 1995). Otro movimiento con una trayectoria importante en el fomento de grupos es el cooperativismo especialmente en la región de Centro Occidental, con la famosa feria de consumo agrícola. Para 1999 existían en el país más de 700 cooperativas con 16 centrales de integración.

\subsection{Década de los 90: La constitución de un nuevo espacio público.}

En la crítica al clientelismo, a la pasividad de la población, a la injerencia de los partidos y del Estado en todos los asuntos de la vida colectiva, se sientan las bases para la construcción de una nueva concepción de ciudadanía, que corresponde a la democracia de los ciudadanos. Sobre esta concepción Lander (2000) señala que tiene como prototipo al ciudadano ligado a la propiedad, que se organiza en defensa de las condiciones privilegiadas de sus espacios urbanos promovida desde el movimiento vecinal, puesto que en sectores donde predomina la pobreza, no puede hablarse de la misma noción de ciudadanía, allí los problemas no se definen por la defensa de lo que se tiene sino por la necesidad de lograr lo que no se tiene. No todos los grupos sociales tienen las mismas condiciones, la desigual dotación de recursos y capacidades, inciden en las iniciativas para crear e imponer determinadas orientaciones.

Cobra auge la tendencia a disminuir las funciones que desempeña el Es- tado, y un refuerzo de la participación del mercado con la incorporación del sector privado en áreas y servicios que antes eran de su competencia., para ello se generaliza una campaña en la cual el Estado es portador de todos los males mientras el resto de la sociedad, es expresión de todas las virtudes, basada en la promoción del modelo de funcionamiento del mercado, En ese mismo sentido, se subestima radicalmente el papel de las instituciones políticas tradicionales y se asumen los supuestos clásicos del pensamiento liberal sobre la separación de lo público y lo privado.

Es en esta coyuntura cuando irrumpen el crecimiento exponencial -en Venezuela- de las ONG's asumiendo la ejecución de políticas y programas sociales, estimulados desde el Estado que ya iniciaba la onda privatizadora.

La Política Social del Estado orientó el diseño de un Programa de Apoyo a la Iniciativas Populares con el objetivo de crear y desarrollar un mecanismo piloto de coparticipación público-privado, para financiar proyectos y actividades formulados y ejecutados por la sociedad civil, instalando en el nivel local capacidades para prestar servicios destinados a los sectores más vulnerables. Se propone“... la organización de la población para su incorporación en la gestión social, promoviendo su participación efectiva en los programas y servicio sociales... para que puedan resolver sus problemas y carencias a través de un esfuerzo solidario y permanente..." (CORDIPLAN: 1990).

Tal y como se viene planteando teóricamente, en América Latina y también en Venezuela el mismo Estado incorpora a las ONG's en sus planes nacio- 
nales (CORDIPLAN, 1990) reconociéndoles aporte para la construcción de ciudadanía, pues se espera de ellas que promuevan en su prácticas el empoderamiento de los sectores más vulnerables, el fortalecimiento de su capacidad de organización indispensable para que los derechos sociales y económicos puedan ser ampliados hacia todos los individuos.

Al respecto, Morales (1998) señala la convergencia entre la tendencia espontánea de la sociedad a autoorganizarse a efectos de obtener los servicios sociales que les falta, y la propuesta que viene del lado del Estado de transferir a Organizaciones No estatales actividades de su exclusividad.

El condicionamiento estatal a las ONG's puede generar las siguientes distorsiones: (Vilas, 2000; Petras, 2000; Ochoa, 2001; Revilla, 2002; Alonso y Jerez 1997)

- Crear un estímulo hacia la profesionalización y la mercantilización del altruismo en la búsqueda y captación de recursos económicos y humanos.

- La dependencia del financiamiento público que las convierte en muchos casos en entidades de subcontratación de servicios para el Estado con la consecuente subordinación a los criterios establecidos por el mismo, con lo cual se desdibujan los elementos diferenciadores de las ONG's con respecto a las organizaciones estatales, como son: iniciativa, flexibilidad, innovación, cambios.

- Flexibilización de trabajo en las propias organizaciones, negando los beneficios contractuales conquistados por los trabajadores y reforzando un abaratamiento del costo del trabajo.
- El reparto de los fondos gubernamentales hacia organizaciones en función de la generación o retribución de lealtades políticas más que de acuerdo al mapa de los grupos con carencias, mantiene y fortalece el clientelismo como práctica de participación.

- Poca transparencia en las asignaciones y ejecuciones presupuestarias por la ausencia de regulación y controles.

- Dependencia respecto a las fuentes de financiamiento externas (Estado u organismos nacionales e internacionales), puede subordinar los intereses de las comunidades a los objetivos del donante y desviar la atención de las causas reales de los problemas de la pobreza.

- Declinar sus responsabilidades de ejercer una función crítica y de control sobre el Estado.

- Competencias no sólo entre las Organizaciones sino también entre los profesionales por la captación de recursos estatales, con lo cual se generan rivalidades internas y externas que socavan la organización popular de base. El ahorro de recursos financieros, a través del trabajo gratuito o pobremente remunerado de los grupos involucrados en los proyectos.

\section{Los inicios del Siglo XXI. Formas de expresión de las ONG's en los Municipio Maracaibo y San Francisco Zulia}

El campo de las ONG constituye un sector sumamente heterogéneo en permanente crecimiento en las últimas déca- 
das; para este estudio se registraron ciento noventa y cinco (195) ONG's orientadas a atender diversos problemas sociales a través de la ejecución de diferentes programas. Esta información se obtuvo con la consulta de las distintas fuentes de información, las cuales incluyeron las instituciones públicas: Gobernación y, Alcaldías entre otros, así como los datos obtenidos a través del Portafolio Social.

Es significativa la expansión del radio de acción y la escala de participación de las ONG's en la prestación de los servicios sociales que se brindan en los municipios, por lo que para su presentación de acuerdo a: Ubicación espacial, ubicación sectorial, instituciones que las promueven, población objetivo, organización y permanencia, y relaciones entre organizaciones e Instituciones.

\subsection{Ubicación espacial}

La mayoría de las organizaciones están ubicadas en el municipio Maracaibo, lo cual es coherente por ser el municipio con mayor población, capital del estado y sede del gobierno regional, sin embargo, es significativo que en el Municipio San Francisco -creado en 1995- exista un $35 \%$ del total.

Esta misma tendencia de concentración se observa en las seis parroquias del Municipio San Francisco, ya que en las más densas en población se concentran el $90 \%$ de las ONG's: San Francisco, Francisco Ochoa y Domitila Flores, a diferencia de las parroquias mas pobres y alejadas de centro urbano: Los Cortijos, Marcial Hernández y el Bajo. Igual situación encontramos en Maracaibo, poca presencia de estas organizaciones en las parroquias más pobres como: San Isidro, Venancio Pulgar, Antonio Borjas Romero e Idelfonso Vásquez.

Esto es un indicador de que las profundas desigualdades sociales inciden en el ejercicio de los derechos ciudadanos, puesto que la organización requiere de actores colectivos, capacidades y recursos, condiciones que -en situación de pobreza extrema- están disminuidas. Vemos así que los grupos sociales mas vulnerables no se constituyen per se en nuevos actores con acceso al espacio socio político y, ello se refleja, en la débil presencia de organizaciones gubernamentales que pudieran ofrecer prestación de servicios en las parroquia mas pobres de ambos municipios donde hay mayor pérdida de acceso a los servicios públicos.

\subsection{Ubicación Sectorial}

Los datos encontrados nos permiten identificar la siguiente ubicación sectorial de servicios: Deportivas y recreacionales, Salud, Educación, Arte y Cultura, Defensa del Ambiente, Hábitat y Vivienda, Desarrollo y Promoción Comunitaria y Benéficas Asistenciales.

\section{Deportivas y recreacionales:}

Este sector constituye el $14 \%$ del universo estudiado. Su crecimiento en este sector es significativo, por el impulso de las Políticas Públicas diseñada por el Instituto Nacional de Deportes (IND), las cuales promueven esta actividad en los sectores populares al ofrecer financiamiento con presentación de proyectos. Estos proyectos se canalizan a través del Instituto Regional de Deporte del Estado Zulia (IRDEZ) -en el programa Deporte para Todos- a través del cual se otorgan los re- 
cursos aprobados por el IND, esto favorece la dependencia financiero de las ONGs. de los recursos del Estado.

Esta orientación de trabajo por proyectos, siguiendo a Petras (1996), contribuye a distanciar a los grupos conformados de los movimientos sociales y los activistas en defensa de los derechos colectivos, pues influye el temor que su participación en las luchas populares les lleve a perder los aportes económicos que reciben del Estado. Por otra parte, la competencia por los escasos recursos perjudica la solidaridad de clase, pues crea distinciones y rivalidades entre las distintas organizaciones y comunidades a las que pertenecen además que desvía la atención de las fuentes y soluciones de la pobreza que los afectan. Por otra parte la dependencia financiera de los recursos del Estado es una de las causas de la poca continuidad en el desarrollo de sus actividades cuando no reciben aprobación de sus proyectos.

Salud: El 18\% del universo de estudio se ubica en el sector salud. En este se destaca como nueva tendencia la creación de Fundaciones al interior de los principales Hospitales públicos de estos municipios y de los Centros Ambulatorios, algunas de las cuales fueron eliminadas con la reorganización de los servicios del sector en referencia y la aplicación de las políticas de salud del gobierno -del presidente Hugo Chávez Frías- que garantizan la gratuidad de estos servicios como es el caso de la Fundación Hospital Universitario, a través de la cual se canalizaban los recursos de las Fundaciones creadas en los distintos servicios del Hospital, cuyo objetivo explicito era de contribuir al mejoramiento y desarrollo de los servicios, pero el establecimiento de tarifas generó discriminaciones entre los distintos usuarios que demandaban la prestación del servicio. lo cual es coincidente a la visión de mercado propuesta por las tendencias neoliberales. Aún se mantienen organizaciones con esta forma de funcionamiento, como es el caso de la Fundación Venezolana para la Medicina Familiar -FUNVEMEFA- que aunque reciben recursos de la Gobernación y empresas del Estado -PDVSA- mantienen el cobro de tarifas de servicios que antes eran gratuitos. Como consecuencia de ello afectan a sectores de la población de escasos recursos, especialmente los vecinos de su sede principal ubicada en Sierra Maestra. Algunos de los usuarios pioneros -con el fallecido Luis Sergio Pérezde la construcción de este centro asistencial, se quejan actualmente de la desviación de los objetivos originales de los socios fundadores, pues no sólo se han mercantilizado los servicios de salud, sino se ha flexibilizado la relación laboral del personal contratado en la misma. Vemos aquí un ejemplo de la contradicción de los intereses corporativos con los intereses comunitarios, (Ochoa, 2001) con lo cual no sólo se acercan al ámbito de lo privado sino que fortalecen la exclusión social considerando la vulnerabilidad económica de los sectores que atienden. Estas formas de atención en salud son coincidentes con la visión de mercado propuesta por la tendencia neoliberal.

En otra dimensión debe destacarse la labor desarrollada por el Hogar Clínica San Rafael dirigida a prestar servicio médico-asistencial a la población infantil, atendiendo de manera preferencial a los sectores sociales más vulnerables; la 
Fundación Innocens cuyo objetivo es brindar atención integral a niños de de bajos recursos del Occidente del país, infectados con VIH-SIDA; el trabajo de la asociación de Alcohólicos Anónimos en la recuperación de personas alcohólicas.

Educación: La prestación de servicios de las ONG's en el área educativa, un $22 \%$ de las estudiadas, es una respuesta por una parte, a uno de los problemas más sentidos en Venezuela, que es la calidad de la educación, especialmente en los sectores sociales de mayor pobreza y, por otra parte, a la ejecución de las políticas sociales focalizadas y de carácter compensatorio la cual contempló delegar -a las organizaciones no gubernamentalesla responsabilidad de ejecutar los programas de Capacitación y Empleo Juvenil dirigido a jóvenes desertores del sistema educativo y Hogares de Cuidado Diario dirigido a niños en edad pre-escolar. Este último programa es coordinado actualmente por la Asociación Civil Hogares de Cuidado Diario -creada por la Gobernación del Estado- a través de la cual se canaliza el traspaso de recursos y supervisiones para aquellas ONG's que prestan servicios en las comunidades a hijos de madres trabajadoras; entre estas podemos mencionar por Ej: Fundación Asociación Civil Organización de Ayuda al Circulo Familiar ORDACIFAM, Amigo de los Niños Marginales. Este programa se ofrece gratuitamente, pero entre sus desventajas está otorgar empleos a las madres cuidadoras en condiciones de flexibilización laboral: muy baja remuneración, trabajo a tiempo parcial, sin prestaciones ni seguridad social. Esto demuestra la tendencia del Estado a disminuir sus funciones, así como compromisos laborales con los trabajadores.
Se considera un programa de carácter muy asistencial para enfrentar el déficit del Estado de Centros Educativos que tiene el Ministerio de Educación para niños en pre- escolar.

Entre las ONG's que se han consolidado no sólo por la atención de los sectores más vulnerables sino por la calidad socialmente reconocida del servicio ofrecido se destaca Fe y Alegría, la cual ha extendido sus servicios educativos a la capacitación laboral de jóvenes desertores del sistema educativo y, mas recientemente, a la Educación Superior con la apertura del Instituto San Francisco. Otras de igual calidad en su trayectoria son las dirigidas a la educación especial como es el caso de la Fundación de Amigos y Representantes de Adolescentes con Retardo Mental (FUNPARM), la Asociación Zuliana de Niños Excepcionales; las que dirigen sus objetivos a la capacitación laboral de jóvenes como es el caso del Equipo de Formación, Información y Publicaciones (EFIP), Asociación Civil Retruque, el Centro La Chinita de la Asociación de Damas Salesianas y el Centro Ángel de la Guarda, entre otros.

Arte y la Cultura. En este sector se ubican un $15 \%$ de las ONGs. Estudiadas. Se caracteriza por una gran heterogeneidad en el tipo de organizaciones, por un lado están aquellas organizaciones tuteladas por el Estado, las cuales reciben financiamiento y subvenciones del Ejecutivo Nacional y Regional como la Fundación Museo Arte de Contemporáneo del Estado Zulia, entre otras y las organizaciones de base impulsadas por líderes arraigados en las comunidades, hacia donde dirigen programas para la difusión de actividades culturales que contribuyan 
a rescatar valores e identidad nacional. Entre estas encontramos por Ej: Fundación “AJE", Grupo de Teatro Tablón, Teatro Esencial, Centro de Información Patrimonial El Tabacal, Asociación Cívica Escena Cultural, Bajo el Sol Colectivo Teatral. En este tipo de actividad se ha dado un sensible crecimiento de ONG's, por la orientación de políticas públicas del Estado, dirigidas por el Consejo Nacional de la Cultura (CONAC), que ofrece apoyo financiero a estas organizaciones con la presentación de proyectos culturales, los cuales son presentados a través de la Secretaría de Cultura del Estado y evaluados para la asignación de los recursos presupuestarios con participación de las mismas organizaciones participantes. Aunque esta modalidad incorpora la participación de los actores participantes en la definición de los presupuestos, no está excepto de poder generar competencia entre los grupos por la captación de los recursos financieros.

Defensa del Ambiente: En las Organizaciones para defensa del ambiente se ha dado un progresivo crecimiento, el $9 \%$ de las ONG's identificadas se ubican en este sector, con presencia de actividades dirigidas a las comunidades, donde se reconocen dos orientaciones, una que van en consonancia con las políticas del Estado a elevar los niveles de conciencia de los ciudadanos a través de la formación de brigadas conservacionistas dirigidas al cuidado de las playas, la siembra y mantenimiento del ambiente, etc. Otra dirigida a desarrollar estrategias alternativas que enfrentan las políticas económicas del Estado que afectan y destruyen el ambiente y el hábitat de grupos humanos, como es el caso de Asociación Civil
Homo et Natura, la cual en acompañamiento de grupos ecológicos e indígenas de la Sierra de Perijá lucha por la no apertura de nuevas minas de carbón, pues contaminan el medio ambiente $y$ desplazan de sus tierras a los grupos indígenas. Ninguna de ellas recibe fondos de dependencias gubernamentales.

Otros sectores: Entre otros sectores se ubica un $22 \%$. Podemos destacar el sector de Hábitat y Vivienda donde una tendencia importante es la formación de ONG's para la presentación de proyectos habitacionales, fundamentalmente, ante los organismos del Estado, concretamente el Consejo Nacional de Vivienda (CONAVI) y recientemente el Ministerio de Hábitat y Vivienda. Se desarrollaron diversos programas con la debilidad del frecuente cambio de reglas y redefinición de los mismos, con lo cual muchas de las ONG's han cesado en sus funciones al no obtener respuestas favorables a sus proyectos.

Las ONG's de Desarrollo y Promoción Comunitaria las cuales desarrollan programas múltiples que incluyen capacitación de lideres, formación de pequeñas empresa, créditos solidarios, entre éstas podemos señalar la Asociación Nuevo Amanecer, el Centro de Orientación Comunitaria $(\mathrm{COC})$ y la Fundación de Apoyo a la Economía Popular (FUDEP) entre otras.

Organizaciones Benéficas Asistenciales: En este sector ubicamos aquéllas que dirigen programas de asistencia social, entre las que podemos señalar la Casa de la Misericordia, Fundación Hogar Santa Ana, Banco de Sillas de Ruedas; Fraternidad Mercenaria Seglar (FRAMERSE). La mayoría de estas ONG's agrupan fundamentalmente muje- 
res que pertenecen a grupos religiosos y se orientan la ideología de amar al prójimo por amor a Dios.

\subsection{Instituciones que promueve las ONG's}

El $35 \%$ de las organizaciones no gubernamentales identificadas se originan por iniciativa eclesial, las cuales cuentan regularmente con óptimas instalaciones de infraestructura y organización administrativa para la prestación de sus servicios, además de que sus miembros cuentan con fuerte liderazgo y capacidad para contactar y movilizar recursos económicos y humanos. Generalmente este grupo de ONG's cuenta con subvenciones del Estado, aportes de socios, tanto en cotizaciones como en prestación voluntaria de trabajo -en muchos casos de jornada completa-, además de aportaciones de empresas privadas e inclusive apoyo internacional.

Dado que la iglesia se identifica como institución de control social por sus propósitos de orientación ideológica, en el caso de las ONG's este propósito está en correspondencia con el compromiso de la jerarquía que incide en la dirección de cada organización, especialmente la aceptación y seguimiento de las ideas fundamentales del Concilio Vaticano II y las Conferencias Episcopales efectuadas en Medellín, Colombia (1968) y Puebla, México (1979): El compromiso con el pueblo. Esto determina la forma de trasmitir algunos valores como: igualdad, equidad, pluralismo, participación pues en la dirección de estas ONG's, encontramos grupos religiosos católicos muy heterogéneos como: Opus Dei, Jesuitas,
Salesianos, Congregaciones de monjas como Las Siervas Pobres de la Madre de Dios y Las Carmelitas, entre otros.

\subsection{Población objetivo}

Encontramos que el porcentajes de menos incidencia se ubica en las ONGs dedicadas a las poblaciones Específicas de Género, Etnia y Ancianos que en conjunto alcanzan sólo un $7 \%$ de las estudiadas.

Las instituciones con más alto porcentaje (40\%) están dedicadas a la Atención de Niños y Jóvenes a través de los distintos programas: recreativos, culturales, educativos y salud implementados por las distintas ONG's citamos por ejemplo: Fundación Animarte y Asociación de Títeres Papagayo, dirigidas al rescate del sentido de pertenencia y los valores tradicionales de niños y jóvenes de la región; Fundación Innocens y Fundación Amigos de niños con cáncer, cuyos objetivos son la atención de niños afectados por SIDA y CANCER respectivamente; La Fundación Peter Alexander y la Asociación Zuliana de Padres y Amigos de Niños Excepcionales (AZUPANE), dedicadas a la atención integral de la población infantil y juvenil con autismo y retardo mental respectivamente; Fe y Alegría y Niños Cantores del Zulia, ofrecen atención integral católica a niños y jóvenes; Asociación Civil, Formación, Información y Publicaciones (EFIP) dedicada a la capacitación laboral de jóvenes; SOS Aldeas Infantiles, Casa Hogar Jardín de Belén y Fundación de Amigos de los niños trabajadores del Zulia Villa Feliz ahora llamada Fundación Sueño Zuliano, las cuales brindan atención integral a niños y adolescentes en si- 
tuación de alto riesgo social; por último, entre otras, se encuentran la Fundación en Pro de la Infancia necesitada y la Fundación Amigos de los niños Marginales que se dedican a la atención de niños en edad pre-escolar hijos de madres trabajadoras.

Con programas dirigidos a las comunidades reconocimos un porcentaje estimado en $31 \%$, lo cual incluye además de las que declaran su objetivo específico $-6 \%$, las de Hábitat y Vivienda -8\%-, Defensa del Ambiente -9\%-, y un grupo de organizaciones del área de cultura -8\%que desarrollan programas dirigidos a las comunidades.

Otro porcentaje de ONG's dirige sus programas a la atención de adultos (22\%) a través de los distintas áreas como el caso de la Asociación de alcohólicos anónimos, de organización como ayuda penitenciaria; en apoyo a los discapacitados como la Asociación Zuliana de Ciegos, Banco de Sillas de Ruedas (BANDESIR); del área salud organizaciones como, Fundación Médico Social Bautista del Zulia.

Del grupo de ONG' vinculadas a la iglesia el $76 \%$, desarrollan programas educativos dirigidos especialmente a niños y jóvenes. En este porcentaje se incluyen grupos cristianos evangélicos, aunque en menor proporción registran un progresivo crecimiento.

\subsection{Organización y Permanencia}

Al amparo de la aplicación del modelo económico de corte neoliberal y el estimable volumen de recursos públicos propuestos para el financiamiento de proyectos sociales, se estimuló la creación de asociaciones y organizaciones que persiguen obtener recursos como estrategias laborales. Esto se constituye en una debilidad por cuanto en un contexto de inestabilidad laboral aumenta las posibilidades de que se aprovechen estos nuevos espacios de trabajo sin poseer las fortalezas conceptuales, metodológicas y la capacidad de gestión necesarias, así como el compromiso social con los sectores mas necesitados, para emprender los objetivos tan complejos que se demandan de ellas, que van desde mejorar la calidad en la atención de los problemas sociales hasta la promoción de cambios socio-culturales que contribuyan a articular el tejido social.

Vemos así como un 14\% de ONG's cesaron en su funcionamiento, muchas de las cuales surgieron al calor de los programas impulsados desde el Estado. Entre este grupo de ONG's se pueden mencionar: Monitores Populares de Derechos Humanos, Fundación Capacitación de Adolescentes y Empleo (Juvenzul), Fundación Michel Lagalla, A.C. Mejoramiento de Viviendas Barrio Virgen del Carmen, Fundación Nueva Vida, Asociación de Jóvenes del Barrio La Caimana, Centro Educativo Popular Doña Ana, Fundación Centro de Capacitación para el Desarrollo Industrial Juvenil y Social. Las principales causas del cese de actividades de estas organizaciones se identificaron con la pérdida del financiamiento del Estado, la debilidad organizativa referida a la concentración de tareas en pocos miembros y la falta de evaluación y control.

Por otra parte, las dinámicas que enfrentan las ONG's activas las llevan a priorizar la atención en la presentación de cuentas y trabajo de carácter cuantitati- 
vos, referidos al manejo de los recursos otorgados de acuerdo a las exigencias de los financistas para lo cual usualmente se enfrentan con limitaciones de conocimientos contables. Esto influye en la insuficiente atención que le asignan a los aspectos cualitativos concernientes a sus compromisos sociales como: Revisión de nuevas opciones de paradigmas, metodológicas, de alianzas así como el debate acerca de los valores que promueven sus formas de gestión, las reformas de las políticas publicas necesarias.

La incorporación de trabajo voluntario, así como la captación de profesionales al equipo de trabajo contribuye a disminuir estas debilidades, pero no todas cuentan con la misma capacidad de movilizar recursos humanos voluntarios $y$ técnicos para integrarlos en la ejecución de sus programas. Las ONG's que generan mayores movilizaciones de recursos son especialmente aquéllas impulsadas desde la iglesia, constituyéndose en una limitación en las que representan colectivos provenientes de los sectores de bajos recursos económicos. A esto se suma la poca práctica y visión de la cooperación, concertación y acuerdos con sus iguales para intentar optimizar sus oportunidades individuales en relación a los recursos y mejorar en el logro de los objetivos planteados y el impacto social de su trabajo.

\subsection{Relaciones entre ONG's}

Otra de las debilidades sentidas de las ONG's es la fragmentación y aislamiento en la ejecución de su trabajo. Poco son los casos de las organizaciones que trabajan en redes o articulando esfuerzos de recursos o tareas, sin embargo, se observó esta tendencia entre las ONG's de capacitación y empleo juvenil del municipio San Francisco y las organizaciones orientadas a la defensa del ambiente. En el municipio Maracaibo se reconoció la ejecución del Programa Ciudadanía Plena por un consorcio entre las Asociaciones Hábitat Luz, la ONG Nuevo Amanecer y la Alcaldía de Maracaibo para la intervención en cinco barrios de esta ciudad con el aporte de una entidad financiera internacional.

En el municipio San Francisco se reconoció la creación de una Red de apoyo entre actores del mismo ámbito sectorial y geográfico, promovida con apoyo de la Universidad del Zulia, a través del equipo de investigación del Centro de Estudio de la Empresa. Esta red identificada como Red de Organizaciones de Capacitación San Francisco (ROCAS) ${ }^{5}$, orienta sus objetivos la coordinación de acciones conjuntas, negociar con otros actores e instituciones públicas propuestas y proyectos a fin de consolidar y mejorar las

5 Esta red se inicio en el año 2001 con la participación de Fe y Alegría, Asociación Civil Equipo de Formación, Información y Publicaciones (EFIP), Asociación Civil Equipo Retruque, Asociación Civil Madre Magdalena Taylor, Centro de Capacitación Ángel de la Guarda, Organización para el Crecimiento Integral Humano (OCIH), Asociación Civil Nuevo Amanecer con el apoyo del Centro de Estudios de la Empresa del la Universidad del Zulia. 
opciones de formación y acceso de los jóvenes a un empleo digno.

\section{Conclusiones}

En los municipios estudiados las ONGs han crecido en las últimas décadas a un ritmo muy superior que las anteriores. Esto, así como las características que han asumido ha sido producto y expresión de los cambios políticos relacionados con el agotamiento de los mecanismos de representatividad política del modelo rentista petrolero -caracterizado por el predominio de relaciones clientelares- que obstaculizaron las tendencias asociativas de la sociedad y a la aplicación de las políticas publicas orientadas por el modelo neoliberal al asumir la ejecución de políticas y programas sociales que desempeñaba el Estado, en la onda privatizadora dirigida a disminuir sus funciones.

Es el análisis de su funcionamiento se hace preciso situarlas en la dimensión que les corresponden, pues no se constituyen en un espacio homogéneo sino atravesado por las luchas sociales y en confrontación entre la lógica estatal de gobernabilidad y la lógica societal centrada en resolución de problemas y de promoción de cambios para la profundización de la democracia. Es por ello que al eliminar la reflexión analítica de los problemas que atienden y, no establecer una clara posición ético-cultural, a través de su desempeño pueden reforzar y justificar el abandono de las responsabilidades del Estado en la lógica del modelo neoliberal, que apunta a objetivos como: el desplazamiento de las funciones del Estado a las organizaciones de la sociedad, la flexibilización laboral, el abaratamiento del costo del trabajo, desconocimiento de los beneficios laborales de los trabajadores, contribución al reemplazo de valores tradicionales por valores del individualismo competitivo, debilitamiento de la capacidad de movilización de actores antagónicos y renovación de vinculaciones clientelares y lealtades políticas antes que a los colectivos.

Es esta la realidad que enfrentan muchas de las organizaciones que se desempeñan en los municipios estudiados, por lo que podemos estar en presencia de esquemas de participación que poco aportan a la meta de profundizar la democracia y contrariamente puedan estar contribuyendo al extender la visión de mercado al convertir a los usuarios en consumidores de determinados servicios no gubernamentales, alejándolos de la reflexión de las causas que generan las problemáticas que atienden y ocupándose primariamente de la búsqueda de recursos y el manejo eficiente de los mismos.

Por otra parte, la debilidad financiera que enfrentan las ONG's puede ubicarlas en una total dependencia del Estado o los entes financistas con lo cual se restringen a ser simples ejecutoras de proyectos, excluyéndolas del debate de la definición y diseño de políticas, las cuales van quedando fuera de la discusión de los colectivos, contribuyendo a legitimar políticas que no han sido consultadas, aún cuando hay un discurso de apertura a la democracia participativa.

La democratización política no es el resultado automático del cambio institucional, pasa por la lucha por enfrentar la concentración de poder, muchas veces enraizada -por tradición cultural- 
en los líderes de estas nuevas asociaciones. Se requiere de formas de organización y participación que susciten en su seno prácticas orientadas al empoderamiento de los sectores mas vulnerables con propuestas con oportunidades de participación efectiva de la mayoría para desarrollar una cultura ciudadana con sentido de responsabilidad social además de ser consecuentes con la recuperación de la función política de discusión ideológica que permita incluir el debate de la influencia de la ideología y modelo neoliberal.

\section{Referencias Bibliográficas}

Alcaldía de Maracaibo y LUZ (1994), Plan de Desarrollo Urbano Local. Documento. Maracaibo. Venezuela.

Alonso, Luis Enrique y Ariel Jerez (1997), "Hacia una politización del Tercer Sector" en ¿Trabajo voluntario o participación? Coordinador Ariel Jerez. Editorial Tecnos S.A. Madrid.

Bresser, Luiz y Nuria Cunill (1998), "Entre el Estado y el mercado, lo publico no estatal". En Lo Público no Estatal en la Reforma del Estado. Editores Bresser P. Luiz C. y Nuria Cunill Grau. Centro Latinoamericano de Administración para el Desarrollo. CLAD. Buenos Aires.

Cernea, Michael M. (1992), "Organizaciones No-Gubernamentales y Desarrollo Local", selección de documentos, CLAD, Vol. 9, No.1, junio. Caracas.

Chossdudovsky, Michel (1979), La Miseria en Venezuela. Editores Vadell Hermanos. Valencia, Venezuela.

CONZUPLAN (1996), Diagnostico Socio-Económico de los Municipios Maracaibo y San Francisco. Gobernación del Estado Zulia. Maracaibo. Venezuela
CONZUPLAN (2000), Características demográficas del Estado Zulia. Gobernación del Estado Zulia. Maracaibo. Venezuela.

COORDIPLAN (1990), El Gran Viraje: Lineamientos Generales del VIII Plan de la Nación.

Covarrubias, Francisco (1992), "Ampliando el concepto de lo no gubernamental". Selección de documentos. Centro Latinoamericano de Administración para el Desarrollo. CLAD. Vol 9, №1. Junio. Caracas.

Defourny, Jacques (1997), "Las vías de la Economía Social No Mercantil y los servicios de proximidad". I Escuela de Verano de Economía Social. En Revista de Debate sobre Economía Pública Social y Cooperativa. CIRIEC No 26 pp 19-41. España.

González, Rosa Amelia, Coordinadora (1998), El sector sin fines de lucro en Venezuela. Cuarto Informe. Equipo de Investigación CISOR. IESA.

Gruson, Alberto et al. (1997), El Polo Asociativo en la Sociedad Civil. Centro de Investigaciones en Ciencias Sociales. Caracas,

Lander, Edgardo (2000), Neoliberalismo, sociedad civil y democracia. Consejo de Desarrollo Científico y Humanístico. UVC.

Lavadenz Mantilla, Fernando (1992), "Las organizaciones No Gubernamentales y los Sistema Locales de Salud." Selección de documentos. Centro Latinoamericano de Administración para el Desarrollo. CLAD. Vol. 9. №1. Caracas. Junio.

Laville, Jean Louis (1999), "La asociación: una organización productiva original”. En Laville,J-L y Sainsaulieu, R. Sociología de las Asociaciones. París. Traducido del francés por Claudia Hernández, revisada por Madeleine Richer. 
Morales, Carlos Antonio (1998), "Suministro de servicio sociales a través de organizaciones públicas no estatales". En Lo público no estatal en la reforma del Estado. Editores Bresser Pereira, Luiz y Nuria Cunill Grau. Centro Latinoamericano de Administración para el Desarrollo. CLAD. Argentina.

Ochoa Henríquez, Haydee (2001), "Descentralización y retiro del Estado de la prestación de servicios en Venezuela". En Revista Ciencias de Gobierno, Año 5, No. 9, Enero-Junio, 2001, 99-121. Instituto Zuliano de Estudios Políticos, Económicos y Sociales (IZEPEZ). Escuela de Gobierno del Estado Zulia.

Petras, James (1996), El postmarxismo rampante: Una crítica a los intelectuales y a las ONG Excelsior, Mexico. 26 de noviembre.

Petras, James (2000), Las dos caras de las ONGs. La jornada, México. 8 de agosto.

Pro Mundi Vita Estudios (1989), "Quien paga la fiesta, toca la orquesta: un reto para la comunidad de las ONG." Periódico bimestral, № 10, julio 1989.

Ramos Rollon, María Luisa (1995), Identidad, Acción y Relevancia Política del Movimiento Vecinal en Venezuela. Nueva Sociedad. Caracas.
Revilla Blanco, Marisa (2002), "Las ONG, la participación política y su relación con el Estado". En: Las ONGs y la política. Ediciones Istmo, S.A. Madrid, España.

Rodríguez, Judith, Rodríguez, Isabel y Scarbay, Lisbeth (2004), "Aproximación a la configuración del tercer sector en los municipio Maracaibo y San Francisco del estado Zulia en el año 2002" Espacio Abierto. Vol. 13 No 2. Universidad del Zulia. Maracaibo. Venezuela.

Salamón Lester y Anheier Helmut K. (1999), Nuevo estudio del sector emergente. Resumen. Proyecto de estudio comparativo del Sector no Lucrativo de la Universidad John Hopkins. Editada por la Fundación BBV. Caracas.

Vargas, Mireya (1999), Las organizaciones de Desarrollo y su acción social en Venezuela. Alianza Social Venamcham. Caracas.

Vilas, Carlos M. (2000), ¿Más allá del Consenso de Washington? Un enfoque desde la política de algunas propuestas del Banco Mundial sobre reforma institucional. Revista Reforma y Democracia No 28. Octubre. CLAD. Caracas. 